\title{
THE BOVINE TYPE OF TUBERCULOSIS ASSOCIATED WITH THREE CASES OF TUBERCULOSIS IN MAN*
}

\author{
MARSHAL FABYAN, M.D. \\ BOSTON
}

Practically all cases of human tuberculosis with which the bovine type of bacillus has been found associated have had their origin in the digestive tract. The invasion takes place either through the mouth or throat into the cervical lymph-nodes, or through the intestinal mucosa into the mesenteric lymph-nodes. Although the infection of cows takes place largely through the air, and first shows itself in the mediastinal or bronchial lymph-nodes, yet there is at present no evidence that adult human beings exposed to the dust of cow-stables contract tuberculosis in this way. The three cases to be briefly reported are also the result of intestinal infection.

\section{REPORTS OF CASES}

CASE 1 (Human No. 251). - For the autopsy notes and material of this case I am indebted to Prof. E. E. Southard, who also supplied the following data concerning the history of the patient:

Patient.-Man, born in Massachusetts, in 1888, of Nova Scotian parents. Became epileptic in 1893. Was admitted to Danvers Hospital in March, 1906; there showed paraplegia, epilepsy and dementia; broke right humerus and right femur in different convulsive attacks, and died Oct. 28, 1908, after a series of convulsions of increasing frequency which developed into status epilepticus last. ing twenty-four hours. During his stay in the hospital the patient drank raw milk daily. His mother began to show signs of pulmonary tuberculosis in 1896 , but was still alive in 1906, when he was admitted.

A utopsy.-The following facts are of interest to us in the autopsy: Body was that of a rachitic individual $120 \mathrm{~cm}$. in length. (Barrel chest, rachitic rosary, anomalies of skull development, and distortion of the long bones.) Sears on the forehead. Pupils unequal, left larger than right.

Mesenteric lymph-nodes swollen and slightly reddened. One near the colon contained a calcitied mass $1.5 \mathrm{~cm}$. in diameter. Chronic perisplenitis.

Pleural cavities show chronic adhesions over the left lower lobe and a persistent thymus.

Lungs free of apical scars; were merely edematous. Lymph-nodes not enlarged.

Heart of normal size. Few sclerotic patches in the coronaries. Slight acute splenitis and fatty liver.

* From the Department of Comparative Pathology, Harvard University Medical School.

1. These numbers correspond to the serial numbers used in the Department of Comparative Pathology, Harvard Medical School, by Prof. Theobald Smith. 
Brain: Weight 1,115 gm. Dura slightly adherent and thickened. Recent fibrinous adhesions to pia. Subpial fluid opaque; convolutions flattened.

Anatomic Diagnosis. - This is given as a questionable acute cerebrospinal men. ingitis with persistent thymus and various chronic conditions, among which may be mentioned chronic adhesive pleuritis; chronic tuberculous lymphadenitis (mesenteric) with calcification; chronic adhesive perisplenitis.

Bacteriologic Cultures.-The mass of lymph-nodes which formed the startingpoint of the cultures was the only manifest tuberculous focus in the body. The nodes were evidently enlarged and permeated with inconspicuous caseous foci. The method usual in this laboratory was resorted to with material of this kind. It was cut up and ground as much as possible in a sterile agate mortar with salt solution and the turbid fluid injected into the peritoneal cavity of two guineapigs. Both became tuberculous, but only one was used for cultures. The method we have found most reliable for coaxing into active growth tubercle bacilli which are cultivated with difficulty is to put bits of tissue as large as peas, usually from the spleen, into Dorset's egg medium, and after from six to eight weeks to spread the masses of growth as' well as the original tissue thoroughly over the surface of fresh egg and glycerin agar tubes. This transfer is repeated until a fairly good growth is obtained on glycerin agar, when the egg medium is abandoned. The other medium most commonly employed-beef serum-is less certain and cultures may be lost. The best medium-dog serum-is obtained with difficulty, and therefore not used at present.

Inasmuch as the morphology of the tubercle bacillus is more or less influenced by the culture medium used, only coagulated serum should be used for comparative studies. On glycerin agar involution forms soon appear to complicate the morphologic picture.

In the present case, after the third transfer on egg medium, representing a total period of growth of over five months, a glycerin agar culture was obtained from which a series of successful subcultures have been carried on. There are at present four tests which taken together enable us to classify mammalian bacilli into the human and the bovine type. These refer to:

1. The form of the bacilli.

2 . The dysgenetic or eugenetic characters of the culture according to the terminology employed by the Royal Commission of England.

3 . The relative virulence toward rabbits.

4. The reaction curve in 5 per cent. glycerin bouillon.

Culture Human 25 was dysgenetic for the usual time, although at present, about eighteen months after isolation, it is nearly as vigorous as the human type. This strain was virulent for rabbits, as indicated in Table 1 , and in its reaction curve it corresponds closely to that of the bovine type, as shown in Table 2. Concerning this table it should be stated that the cultures there given were chosen because they were prepared simultaneously from the same lot of bouillon and were made in every respect under identical conditions.

CASE 2 (Human No, 26).-For the clinical history and the autopsy notes I am indebted to Dr. L. J. Rhea of the Department of Pathology of the Harvard Medical School.

Patient.-The case was that of an infant. Up to February, 1909, there had been no illness. At that time an acute but slight illness appeared, thought to be bronchitis. Thereafter the child was only comparatively well, but at no time actually ill. Temperature records are lacking. Death occurred in April, after symptoms of acute nephritis. There was no source of the tuberculous infection traceable to the immediate environment of the child. The autopsy in this case is definite and perhaps the anatomic diagnosis will be sufficient. 

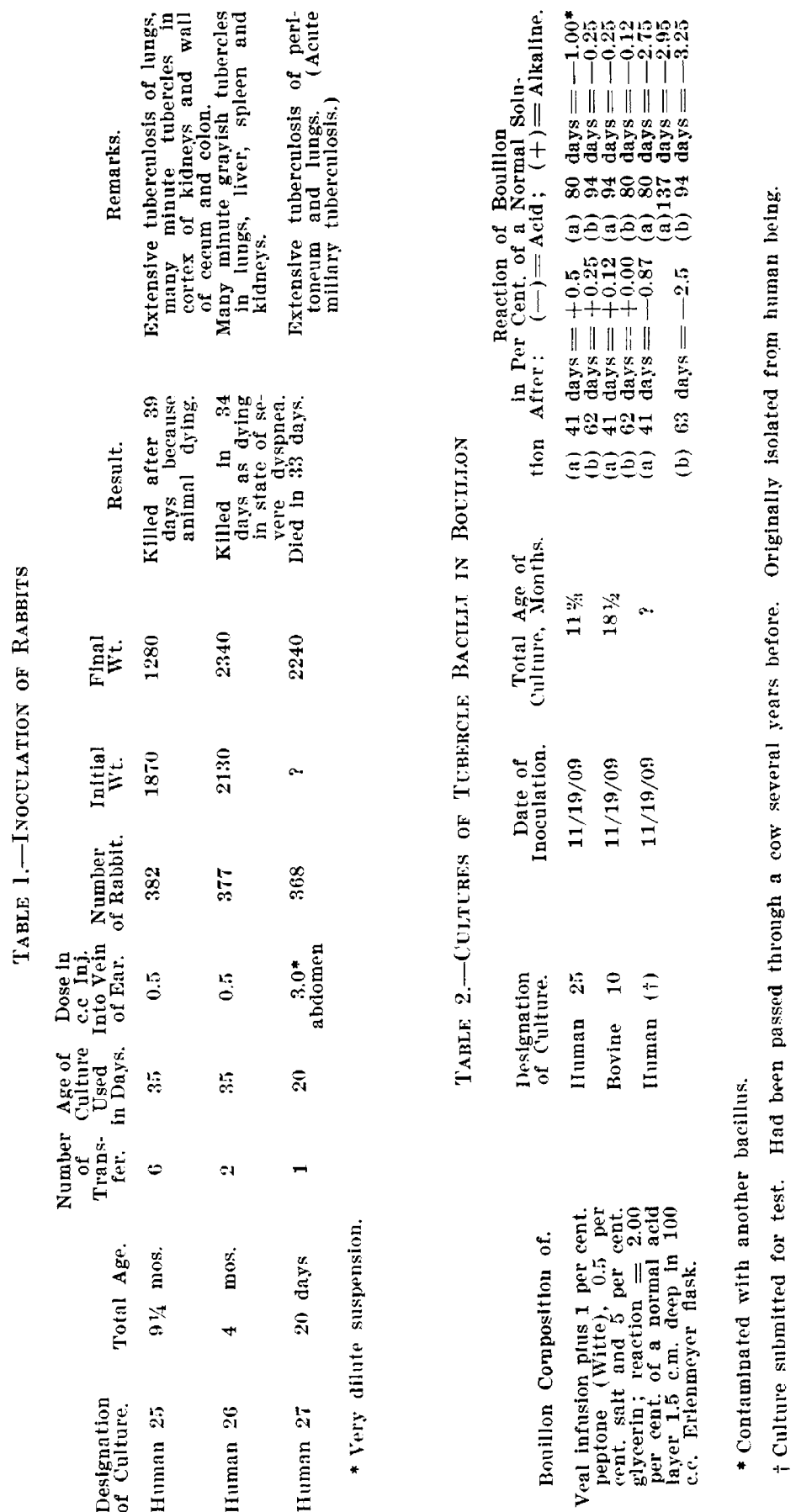

Downloaded From: http://archinte.jamanetwork.com/ by a University of Arizona Health Sciences Library User on 06/03/2015 
Anatomic Diagnosis.-Tuberculosis of the intestines and mesenteric lymphnodes; tuberculosis of lungs, spleen, liver, and peritoneum; involvement of the bronchial glands. Judging by the stage of the disease, the process was oldest in the intestines, where there was ulceration, and in the mesenteric lymph-nodes, which contained creamy areas of softening. In the other organs the lesions had not advanced beyond the small discrete or confluent grayish, somewhat translucent, tubercles.

Bacteriologic cultures.-The culture was isolated from a completely caseous, softened mesenteric lymph-node by passage through three guinea-pigs. Cultures were isolated from each guinea-pig in the manner indicated above, and all but one strain discarded after a time. The feeble growth on glycerin agar has continued up to the present, so that the reaction curve in glycerin bouillon has not yet been obtained, although the strain has been just one year under cultivation. Its virulence for rabbits is shown in Table 1. Altogether the strain must be regarded as of bovine origin.

CASE 3 (Human No. 27). - This strain has been under cultivation only a short time, but I venture to class it with the bovine type because of its very short form and its high virulence for rabbits.

Patient.-The clinical history of the case is as follows: A previously healthy baby girl had a convulsion when 16 months old. The attack was preceded by a few days of malaise. The child recovered slowly, showing some weakness of the limbs on the left side. Ten days later there was a second convulsion, the child remaining in a more or less comatose state until death, about a month from the first symptoms. The signs pointed to a meningitis, and a probable diagnosis of tuberculous meningitis was made. Lumbar puncture was performed and 30 c.c. of clear fluid obtained. An examination of the cellular elements of this fiuid showed a marked increase of lymphocytes (85 per cent). The child died in a few days.

Autopsy.-The notes, for which I am indebted to Prof. E. E. Southard, were apparently quite negative with the exception of the brain.

The mesenteric lymph-nodes were described as numerous, fairly large, and in part considerably injected, but no node showed caseation or other focal lesion. Smears from these glands, however, did show tubercle bacilli.

Pleural cavities free from adhesions. Lungs congested; possibly an early pneumonic process. Bronchial lymplh-nodes not remarkable.

'onsils not obtained. No lesion noted clinically.

The brain substance bulged on removal of the calvarium. A thick exudate containing numerous tubercles covered the base. The pia mater contained numer. ous miliary and conglomerate tubercles having a rather characteristic vascular distribution. The prefrontal and the inferior parietal and occipital regions were freest of tubercles, the right inferior-frontal being most involved.

Anatomical Diagnosis.-Acute edema and congestion of the Jungs; slightly injected mesenteric lymph-nodes; tuberculous meningitis.

Bacteriologic Cultures.-The material from which the cultures were ultimately obtained was the spinal fluid withdrawn during life by lumbar puncture and injected into the peritoneal cavity of two guinea-pigs. From these, cultures on egg media were made in the usual way. The growth was very slow, but after about seven weeks colonies were distinct, and a smear showed very short forms not exceeding one micron in length. As indicated in Table I, a rabbit was inoculated with one of the first cultures from a guinea-pig on egg medium. At the date of the injection the growth was so feeble that in a smear from a suspension in normal salt solution only two clumps of bacilli were detected. A relatively large amount of this suspension was therefore injected into the peritoneal cavity 
at a venture. The rapid death of the rabbit, the presence of myriads of tubercles on all peritoneal surfaces and a well-advanced generalized embolic tuberculosis of the lungs demonstrated a high virulence for rabbits. I do not hesitate, therefore, to range this culture with the bovine type, although a study of it is only partly under way.

These three cases illustrate three different types of tuberculosis, all obscure as to diagnosis during life. In the first case-that of an adolescent-either the local tuberculosis may have had no immediate connection with the cause of death, or else the beginning of dissemination of the virus from the primary focus may have led to a reaction of the tuberculin type and to death. In the second case-that of an infantthe generalized tuberculosis starting from the mesenteric lymph-nodes was evidently not suspected, for the clinical history states that within the last week of life the patient had an acute illness of an indefinite type, which culminated in symptoms of acute nephritis. Here also the liberation of tubercle toxins may have been responsible for the acute nephritis.

In the third case-that of a child of seventeen months-the symptoms pointed to the central nervous system and at one time a tentative diagnosis of anterior poliomyelitis was made.

In conclusion it should also be stated that no similar cases have come under observation during this period. They may be considered three consecutive cases as studied in this laboratory, all due to the bovine type of tubercle bacillus. 